\title{
Metal-Free Alpha Trifluoromethylselenolation of Carbonyl Derivatives under Batch and Flow Conditions
}

\author{
Elisabetta Massolo ${ }^{1}$, Margherita Pirola ${ }^{1}$, Sergio Rossi ${ }^{1, * \mathbb{C}}$ and Tiziana Benincori ${ }^{2, *}$ \\ 1 Dipartimento di Chimica, Università degli Studi di Milano, Via Golgi 19, 20133 Milano, Italy; \\ elisabetta.massolo@unimi.it (E.M.); margherita.pirola@unimi.it (M.P.) \\ 2 Dipartimento di Scienza e Alta Tecnologia, Università degli Studi Dell'Insubria, Via Valleggio 11, \\ 22100 Como, Italy \\ * Correspondence: Sergio.rossi@unimi.it (S.R.); tiziana.benincori@uninsubria.it (T.R.); \\ Tel.: +02-5031-4166 (S.R.); +031-238-6615 (T.R.)
}

Academic Editor: Maurizio Benaglia

check for Received: 16 January 2019; Accepted: 14 February 2019; Published: 18 February 2019

\begin{abstract}
Trifluoromethylselenolated carbonyl compounds represent an emerging class with potential applications in several fields; however, a widespread use of such compound is hampered by the very limited number of strategies for their preparation. In this study we developed a method for the preparation of $\alpha-\mathrm{SeCF}_{3}$ substituted carbonyl derivatives using an in situ generated electrophilic $\mathrm{ClSeCF}_{3}$ species. We also implemented an in-flow protocol to improve the safety features of the process.
\end{abstract}

Keywords: trifluoromethylselenolation; carbonyl compounds; selenium; fluorinated derivatives; flow chemistry

\section{Introduction}

Selenium containing compounds have been shown to be relevant in a number of different areas ranging from health sciences to nanotechnology, through organic synthesis. Selenium has been included in drug design [1], Ebselen being an outstanding example, which is endowed with anti-oxidant, anti-inflammatory, cytoprotective activity, and with potential applications in the treatment of mental disorders [2,3].

Organoselenium derivatives have been appreciated as constituents of self-assembled monolayers [4] substrates used in novel transformations, chiral and achiral organocatalysts, and ligands [5-7]. In recent years, new structural units rising from the association between chalcogens and fluorinated moieties have been introduced and studied for their unique properties. Several new reagents have been designed as a source of electrophilic $\mathrm{SCF}_{3}$, and a scale of their relative reactivities has been established [7]. However, most of them lack a selenium-containing analogue [8] and, as a consequence, the construction of the $\mathrm{C}-\mathrm{SeCF}_{3}$ bond has been rarely reported [9]. Similar to the preparation of highly reactive and toxic halogenated trifluoromethylthio derivatives, the in situ generation of trifluoromethylselenochloride species has been developed using different approaches. In particular, in the late 1950s, Emeléus performed the chlorination of bis(trifluoromethyl)diselenide to yield $\mathrm{ClSeCF}_{3}$ starting from trifluoroiodomethane and selenium at $260-285{ }^{\circ} \mathrm{C}$ [10]. One year later, Yarovenko reported the preparation of hexafluorodimethyl diselenide from trifluoroacetic acid salts (Scheme 1a) [11]. In 2002, Magnier and Wakselman reported the reaction between dibenzyldiselenide and trifluoromethyliodide to afford benzyl (trifluoromethyl)selenide that after treatment with sulfuryl chloride afforded $\mathrm{ClSeCF}_{3}$ in $85 \%$ overall yield (Scheme $1 \mathrm{~b}$ ) [12]. In a more recent approach, Billard, 
inspired by the work of Wakselman, developed a convenient strategy for the generation of $\mathrm{ClSeCF}_{3}$ starting from benzyl trifluoromethyl selenide $\left(\mathrm{BnSeCF}_{3}\right)$ and $\mathrm{SOCl}_{2}$ (Scheme 1c) [13].

a) Emeléus and Yarovenko work

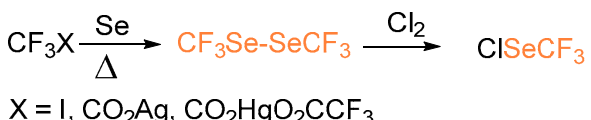

b) Magnier and Wakselman work

BnSe-SeBn $\stackrel{\mathrm{CF}_{3} \mathrm{I} / \mathrm{HOCH}_{2} \mathrm{SO}_{2} \mathrm{Na}}{\longrightarrow} \mathrm{BnSeCF}_{3} \stackrel{\mathrm{SO}_{2} \mathrm{Cl}_{2}}{\longrightarrow} \mathrm{CISeCF}_{3} \mathrm{O}$

c) Billard work

$\mathrm{BnBr} \stackrel{\mathrm{KSeCN}}{\longrightarrow} \mathrm{BnSeCN} \underset{\mathrm{TBAF}}{\stackrel{\mathrm{TMS}^{-\mathrm{CF}_{3}}}{\longrightarrow}} \mathrm{BnSeCF}_{3} \stackrel{\mathrm{SO}_{2} \mathrm{Cl}_{2}}{\longrightarrow} \mathrm{CISeCF}_{3}$

Scheme 1. Different approaches for the synthesis of trifluoromethylselenochloride.

$\mathrm{ClSeCF}_{3}$ is one of the most widely used reagents used to introduce a $\mathrm{SeCF}_{3}$ group into organic molecules, a topic of great interest, as demonstrated by the recent progress toward trifluoromethylselenolation reactions $[9,14,15]$. Moreover, the high lipophilicity and high electron-withdrawing character of $\mathrm{SeCF}_{3}$ group (Hansch lipophilicity parameter $\sigma_{\mathrm{R}}=1.29$ compared to $\pi_{\mathrm{R}}=0.88$ for $\mathrm{CF}_{3}$ group) [16], make it a potential candidate for pharmaceutical and agrochemical applications. As part of our continuing interest in the development of $\alpha$-chalcogen functionalization of carbonyl compounds [17-19] we report herein our study on the introduction of the $\mathrm{SeCF}_{3}$ group on enols of various natures, using $\mathrm{ClSeCF}_{3}$ as the trifluoromethylselenolating agent. In our approach, we decided to take advantage of the straightforward strategy developed by the Billard's group for the in preparation of the highly reactive $\mathrm{ClSeCF}_{3}$ reagent [20] and studied both the in-batch and the unprecedented in-flow transformation.

\section{Results and Discussion}

At first, we investigated the $\alpha$-trifluoromethylselenolation of acetoacetone 1a with the in situ generated $\mathrm{ClSeCF}_{3}$ species (Scheme 2). After some preliminary investigations (see Supporting Information), we established the standard reaction conditions to be the following: $18 \mathrm{~h}$ at room temperature, dichloromethane as solvent, 1.5 molar equivalents of $\mathrm{SO}_{2} \mathrm{Cl}_{2}$, and a threefold excess of the chosen nucleophile.

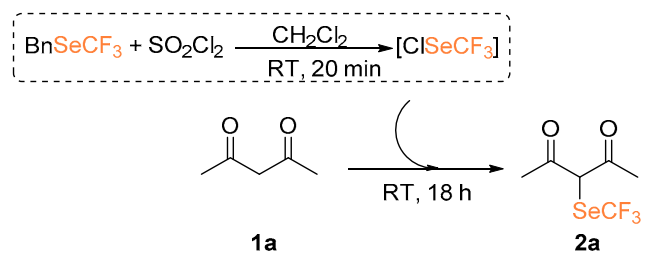

Scheme 2. Attempted $\alpha$-trifluoromethylselenolation of acetoacetone.

Despite the fact that we observed the formation of compound 2a, its isolation led to extremely low yields; this could be due to an increased volatility of the reaction product induced by the introduction of the $\mathrm{SeCF}_{3}$, in analogy to what has been previously observed in our group in the case of trifluoromethylsulfonylation of ketones [16]. We tested the possibility of functionalizing 3-methyl-acetoacetone $\mathbf{1} \mathbf{b}$, thus generating a quaternary center (entry 1, Table 1). Also in this case, ${ }^{19} \mathrm{~F}$ NMR analysis performed on the crude mixture suggested the presence of the properly functionalized diketone $\mathbf{2} \mathbf{b}$. However, purification and product isolation were unsuccessful. No product isolation was 
possible even with products derived from 1-pheny-1,3-butandione 1c and 1,3-indandione 1d although congruent NMR signals were identified in the crude mixture [21].

Table 1. $\alpha$-Trifluoromethylselenolation of diketones.

Entry Reagent Desired Product

The reactions of $\beta$-ketoesters and $\beta$-diester as nucleophiles were also investigated (Scheme 3). Using benzyl acetoacetate (3a) the desired product $4 \mathbf{a}$ was obtained in $16 \%$ yield. With the $\alpha$-substituted ethyl 2-methyl-3-oxobutanoate $3 \mathbf{b}$ and ethyl 2-benzyl-3-oxobutanoate 3c, functionalization occurred at both $\mathrm{C} 1$ and $\mathrm{C} 3$ (both products $\mathbf{4 b - 4 c}$ and $\mathbf{4} \mathbf{b}^{\prime} \mathbf{-} \mathbf{4} \mathbf{c}^{\prime}$ were obtained). Although this does not represent a synthetically useful outcome, it highlights the extreme reactivity of this trifluoromethylselenolating agent: despite the steric hindrance on the more reactive $\mathrm{C} 3$ hampering its functionalization, the reaction still proceeds and the less acidic $\mathrm{C} 1$ is finally involved in $\mathrm{C}-$ Se bond formation.

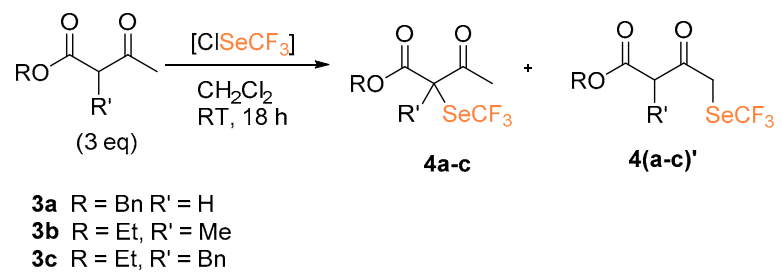

Scheme 3. $\alpha$-Trifluoromethylselenolation of $\beta$-keto esters.

Diethylmalonate $\mathbf{5 a}$ and dimethyl methylmalonate $\mathbf{5 b}$ were also subjected to the metal-free trifluoromethylselenolation protocol, in the presence of in situ generated $\mathrm{ClSeCF}_{3}\left(\mathrm{Scheme}_{4}\right)$. In this case, the desired selenolated product 6 a was isolated in $44 \%$ yield. The use of triethylamine as a base to trigger enolate formation was revealed to be detrimental since no formation of the desired product was observed. Functionalization of $\mathbf{5 b}$ for the generation of a quaternary carbon led to the product $\mathbf{6 b}$, albeit in low yield.

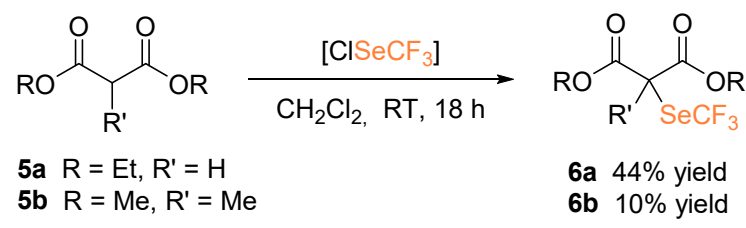

Scheme 4. $\alpha$-Trifluoromethylselenolation of malonates.

We then focused our attention on the $\alpha$-trifluoromethlyseleno derivatization of simple ketones 7a-f. Aromatic and aliphatic ketones with different substitutions were investigated in the metal-free $\alpha$-trifluoroseleno functionalization by reaction with $\mathrm{ClSeCF}_{3}$ species (Scheme 5). 
Under these conditions, trifluoromethylselenated compound 8a, derived from propiophenone $\mathbf{7 a}$ was isolated in $80 \%$ yield. However, the presence of either an electron donating or withdrawing group on the aromatic ring caused a decrement in terms of yield, as demonstrated by the lower yields obtained using compounds $7 \mathbf{b}$ and $\mathbf{7 c}$. Steric hindrance plays a fundamental role in the $\alpha$-trifluoroseleno functionalization: under standard experimental conditions, the reaction of 2,4,6-trimethylacetophenone $7 \mathbf{d}$ leads to the formation of desired $\mathbf{8 d}$ in $11 \%$ yield only. Aromatic cyclic ketones such as 2-acetonaphthone $7 \mathrm{e}$ and 1-indanone $7 \mathrm{f}$ afforded the corresponding $\alpha$-trifluoromethylselenolated compounds $8 \mathbf{e}$ and $8 \mathrm{f}$ in $14 \%$ and $30 \%$ yield, respectively. In all these cases, purification via column chromatography enabled isolation of the desired product, and in addition chlorinated by-products 9 and unreacted starting material [22].

The use of less reactive aliphatic ketones such as cyclohexanone $\mathbf{7 g}$ led to the formation of the corresponding product $8 \mathrm{~g}$ in a yield lower than $16 \%$. The trifluoromethylselenolated derivative of propiophenone (8h) was isolated in $45 \%$ yield. Attempts to functionalize aldehyde substrates such as hydrocinnamic aldehyde $\mathbf{1 0}$ were also performed, but the desired product $\mathbf{1 1}$ was isolated in less than $9 \%$ yield.
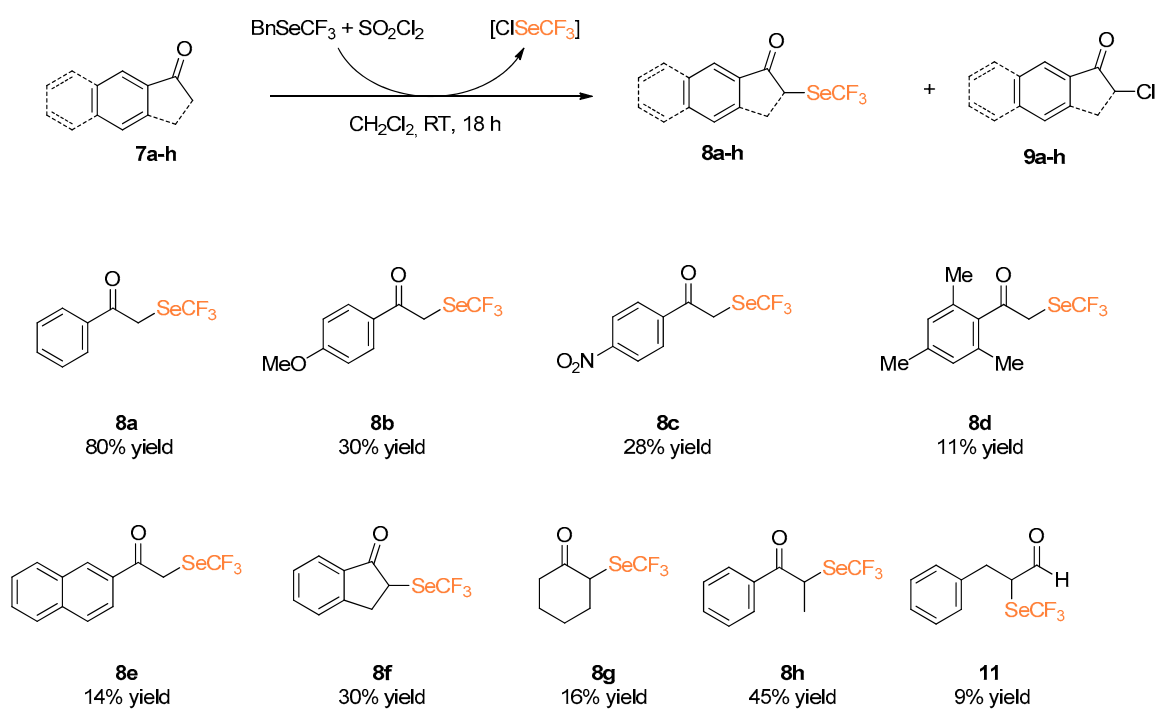

Scheme 5. $\alpha$-Trifluoromethylselenolation of ketones, enamines, silyl enol ethers and aldehydes.

The modest to low yields were most likely due to suboptimal experimental conditions. Indeed, the reactions were performed on a very small scale, as proof of concept of the possibility to realize such transformations, while full information about the toxicity of these selenium-containing compounds is not available yet.

We reasoned that, to improve the safety aspects, the implementation of an in-flow version would have been a valuable alternative. Our aim was thus to set the basis for scale up under continuous conditions, generating and trapping the low boiling $\mathrm{ClSeCF}_{3}$ species in a confined environment. A modular system was conceived where, at the end of a first section of hosting the reaction between $\mathrm{BnSeCF}_{3}$ and $\mathrm{SO}_{2} \mathrm{Cl}_{2}$ (PTFE coil, $40 \mu \mathrm{L}$ volume with internal diameter of $0.07874 \mathrm{~cm}$ ) the nucleophile's inlet would be inserted and the $\alpha$-derivatization would occur in a second reactor section (PTFE coil, $20 \mu \mathrm{L}$ volume with internal diameter of $0.07874 \mathrm{~cm}$ ) (Figure 1). In building the flow reactor, material compatibility needs to be evaluated first; as chlorine is evolved, polytetrafluoroethylene (PTFE) is required [23]. With the available equipment, we realized a mesoreactor small enough to avoid the handling, accumulation, and production of selenolated substances. Considering such a small reaction volume ( $60 \mu \mathrm{L}$ total volume), low flow rates were needed to allow an adequate residence time.

On the basis of preliminary observations, the effectiveness of the generation of the $\mathrm{ClSeCF}_{3}$ could be achieved by running the first step at a temperature slightly higher than RT. Flow rate and reactants concentrations were progressively optimized. In particular, we observed that $\mathrm{SO}_{2} \mathrm{Cl}_{2}$ could not be 
added neat due to its high density hampering effective addition under our conditions of low flow rates. Besides, a tetrahydrofuran (THF) solution revealed unsuitable, as this solvent probably promotes its deactivation before entering the reaction mixture.

With propiophenone as a model nucleophile, conversion to the desired product improved from $70 \%$ to $>99 \%$, when raising the temperature of the first step from 37 to $41{ }^{\circ} \mathrm{C}$. An increase in the temperature, however, causes a higher gas generation and induces irregular discontinuities in the reaction flow, thus negatively affecting the process reproducibility. To limit the occurrence of this hard to control microfluidics alteration without excessively hindering the redox step, we set $37^{\circ} \mathrm{C}$ as an acceptable compromise. The applied addition rate was the smallest possible to allow a sufficient residence time without altering flow regularity. Optimized reaction conditions are shown in Figure 1; stationary conditions were achieved after a minimum of four reaction volumes.

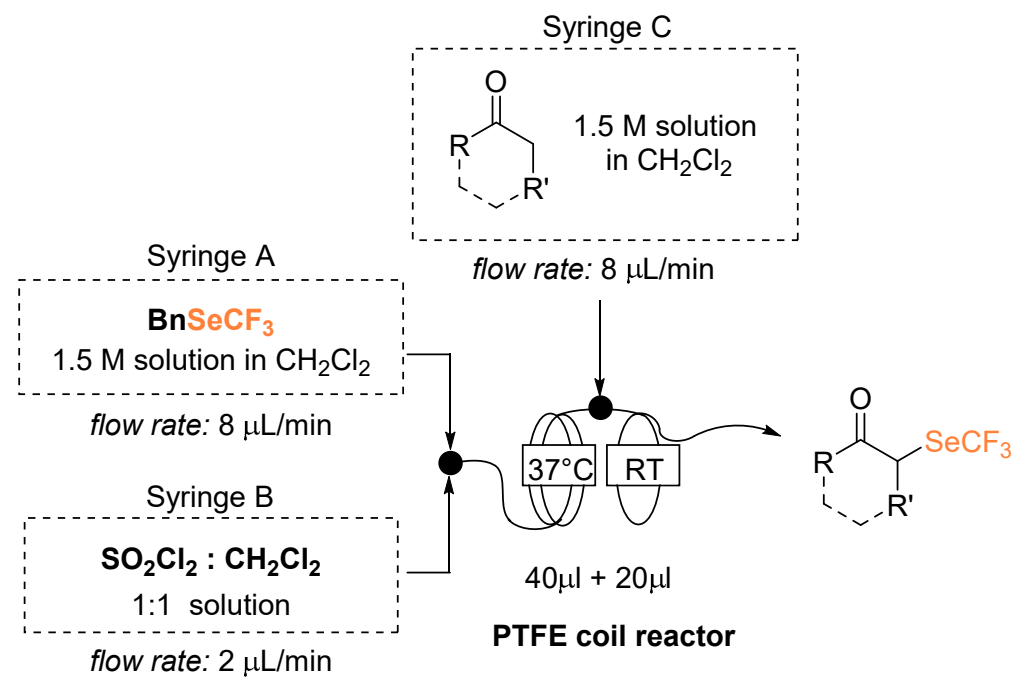

Figure 1. $\alpha$-Trifluoromethylselenolation under flow conditions. Reaction temperature 1 st step: $37^{\circ} \mathrm{C}$, 2nd step: RT. Flow rate inlet A and C: $8 \mu \mathrm{L} / \mathrm{min}$, inlet B: $2 \mu \mathrm{L} / \mathrm{min}$; total flow rate: $18 \mu \mathrm{L} / \mathrm{min}$. Residence time 1st reactor $4 \mathrm{~min}$; 2 nd reactor $1.1 \mathrm{~min}$; total residence time $5.1 \mathrm{~min}$.

With these optimized parameters, trifluoromethyl selenolated derivatives were obtained from acetophenone and cyclohexanone in $70 \%$ and $80 \%$ NMR yield, respectively (Table 2, ketones added neat). When using $p$-methoxy- and $p$-nitroacetophenone ( $7 \mathbf{b}$ and $7 \mathbf{c}$ ), side reactions occurred leading to fluorinated by-products [24]. We rationalized this result by considering slower C-Se bond formation, possibly due to the solid ketones being added as solutions [25]. We reasoned the formation of $\mathrm{ClSeCF}_{3}$ was not completed in the first reactor and was pushed forward by its trapping by the nucleophile; therefore, when an inefficient enolate attack occurs, unreacted $\mathrm{BnSeCF}, \mathrm{ClSeCF}_{3}, \mathrm{SO}_{2} \mathrm{Cl}_{2}$ and its decomposition products coexist for a longer reaction time and undertake undesired pathways. This trend was confirmed when using the poorly nucleophilic diethylmalonate. With $\beta$-ketoester 3a, instead, clean $44 \%$ conversion to the selenolated product was observed.

Table 2. $\alpha$-Trifluoromethylselenolation of ketones performed under flow conditions.

\begin{tabular}{ccc}
\hline Entry & Substrate & ${ }^{\mathbf{1 9}}$ F-NMR Conversion $(\%) \mathbf{~}^{\mathbf{1}}$ \\
\hline 1 & $3 \mathbf{a}$ & 44 \\
2 & $7 \mathbf{a}$ & 70 \\
$3^{2}$ & $7 \mathbf{b}$ & 41 \\
$4^{2}$ & $\mathbf{7 c}$ & 23 \\
5 & $\mathbf{7 g}$ & 80 \\
$6^{3}$ & $\mathbf{7 h}$ & 70 \\
\hline
\end{tabular}

${ }^{1}$ Calculated using PhCOCF 3 as internal standard. ${ }^{2}$ [ketone $]=2 \mathrm{M}$ in dry $\mathrm{CH}_{2} \mathrm{Cl}_{2} \cdot{ }^{3}\left[\mathrm{BnSeCF}_{3}\right]=1 \mathrm{M}$ in dry $\mathrm{CHCl}_{3}$. 


\section{Materials and Methods}

${ }^{1} \mathrm{H}$ Reactions were monitored by analytical thin-layer chromatography (TLC) using silica gel $60 \mathrm{~F}_{254}$ pre-coated glass plates $(0.25 \mathrm{~mm}$ thickness $)$ and visualized using UV light. Flash chromatography was carried out on silica gel (230-400 mesh). Proton NMR spectra were recorded on spectrometers operating at $300 \mathrm{MHz}$ (Bruker Fourier 300, Billerica, MA, USA); proton chemical shifts are reported in ppm $(\delta)$ with the solvent reference relative to tetramethylsilane (TMS) employed as the internal standard $\left(\mathrm{CDCl}_{3}, \delta=7.26 \mathrm{ppm}\right) .{ }^{13} \mathrm{C}-\mathrm{NMR}$ spectra were recorded on $300 \mathrm{MHz}$ spectrometers (Bruker Fourier 300) operating at $75 \mathrm{MHz}$, with complete proton decoupling; carbon chemical shifts are reported in ppm ( $\delta$ ) relative to TMS with the respective solvent resonance as the internal standard $\left(\mathrm{CDCl}_{3}, \delta=77.0 \mathrm{ppm}\right) .{ }^{19} \mathrm{~F}-\mathrm{NMR}$ spectra were recorded on $300 \mathrm{MHz}$ spectrometers (Bruker Fourier 300 ) operating at $282.1 \mathrm{MHz}$; fluorine chemical shifts are reported in ppm $(\delta)$ relative to $\mathrm{CF}_{3} \mathrm{Cl}$ with the respective solvent resonance as the internal standard $\left(\mathrm{CDCl}_{3}, \delta=77.0 \mathrm{ppm}\right)$. Mass spectra and accurate mass analyses were carried out on a VG AUTOSPEC-M246 spectrometer (double-focusing magnetic sector instrument with EBE geometry) equipped with EI source or with LCQ Fleet ion trap mass spectrometer, ESI source, with acquisition in positive ionization mode in the mass range of $50-2000 \mathrm{~m} / z$. The high volatility of the products did not always allow the residence of the sample in the source for a long enough time to record high-res spectra; in these cases, the mass of the products was nevertheless checked by low-resolution mass experiment. The fluidic device was realized by assembling one coil-reactor; reactors were connected, according to Figure 1, by T-junctions using standard HPLC connectors. Coil-reactors consisted of PTFE tubing (diameter: $0.07874 \mathrm{~cm}$ ) coiled in a bundle. Syringe pump: Chemix Fusion 100, equipped with two $1 \mathrm{~mL}$ Hamilton gastight syringes.

\subsection{General Procedure for the Metal-Free $\alpha$-Trifluoromethylselenolation of Carbonyl Derivatives under Batch Conditions}

Benzyltrifluoromethyl selenide ( $0.1 \mathrm{mmol}, 1.0$ equivalents) was dissolved in dry $\mathrm{CH}_{2} \mathrm{Cl}_{2}(100 \mu \mathrm{L}$, $1 \mathrm{M})$ and $\mathrm{SO}_{2} \mathrm{Cl}_{2}(14 \mu \mathrm{L}, 1.5$ equivalents) was added. The reaction mixture was stirred at $\mathrm{RT}$ for $20 \mathrm{~min}$, then the nucleophile ( 3 equivalents) was added. The resulting mixture was stirred for $18 \mathrm{~h}$. The reaction was then partitioned between a saturated water solution of $\mathrm{NH}_{4} \mathrm{Cl}$ and $\mathrm{CH}_{2} \mathrm{Cl}_{2}$, and the aqueous phase was extracted with $\mathrm{CH}_{2} \mathrm{Cl}_{2}$. The combined organic phases were dried over $\mathrm{Na}_{2} \mathrm{SO}_{4}$. The solvent was removed under moderate vacuum and the crude was analyzed via ${ }^{1} \mathrm{H}-\mathrm{NMR}$ and ${ }^{19} \mathrm{~F}-\mathrm{NMR}$. The crude was purified by flash column chromatography on silica gel eluting with the proper pentane $/ \mathrm{Et}_{2} \mathrm{O}$ mixture. The desired product was isolated and the solvent removed by flushing air.

\subsection{General Procedure for the Metal-Free $\alpha$-Trifluoromethylselenolation of Carbonyl Derivatives under Flow Conditions}

The flow reactor was fed by a continuous flow of reagents and reactants: Hamilton gastight syringes were loaded with the needed starting material, whose addition at the defined constant rate was guaranteed by syringe pumps. A $1.5 \mathrm{M}$ solution of (trifluoromethyl)selanylbenzene in $\mathrm{CHCl}_{3}$ was loaded in syringe $\mathrm{A}$; a $6.2 \mathrm{M}$ solution of $\mathrm{SO}_{2} \mathrm{Cl}_{2}$ in $\mathrm{CHCl}_{3}$ was loaded in syringe $\mathrm{B}$; the ketone (neat when liquid, as a solution in $\mathrm{CHCl}_{3}$ when solid) was loaded in syringe $\mathrm{C}$. The first flow reactor (PTFE tube, $40 \mu \mathrm{L}$ volume) was kept at $37^{\circ} \mathrm{C}$ in a thermostated water bath; the second flow reactor (PTFE tube, $20 \mu \mathrm{L}$ volume) was kept at room temperature. The reaction mixture flow was collected on a silica pad placed at the exit of the reactor.

\section{Conclusions}

In conclusion, the $\alpha$-trifluoromethlyseleno functionalization of carbonyl derivatives was studied, using the in situ generated highly reactive species $\mathrm{ClSeCF}_{3}$. A safe and simple experimental procedure was applied for the straightforward introduction of a $\mathrm{SeCF}_{3}$ unit in $\alpha$-position to a carbonyl group. Investigating different carbonyl derivatives, modest to good yields were obtained. For the first time, a continuous process implemented in a tailor-made modular flow reactor allowed the reaction to be 
run under controlled conditions, improving the safety of the process. Despite further studies needing to be undertaken to improve the synthetic utility and to widen the reaction scope, this approach already allows the preparation of highly versatile building blocks, useful as starting material for further synthetic elaborations, with potential for application in several fields, as well as opening the pathway to the discovery of novel products of pharmaceutical interest.

Supplementary Materials: Characterization and purification of compounds synthesized in the text is reported in details in the Electronic Supplementary Material.

Author Contributions: Methodology and experimental work, E.M. and M.P.; conceptualization, S.R.; supervision, T.B.

Funding: S.R. thanks Università degli Studi di Milano for Research grant: Piano Sostegno alla Ricerca—PSR2017.

Acknowledgments: E.M. thanks Università degli Studi di Milano for a postdoctoral fellowship.

Conflicts of Interest: The authors declare no conflict of interest.

\section{References and Notes}

1. Carland, M.; Fenner, T. ${ }^{34}$ Se The Use of Selenium-Based Drugs in Medicine. In Metallotherapeutic Drugs and Metal-Based Diagnostic Agents: The Use of Metals in Medicine; Gielen, M., Tiekink, E.R.T., Eds.; Wiley: Hoboken, NJ, USA, 2005; pp. 313-332.

2. Schewe, T. Molecular actions of ebselen-an antiinflammatory antioxidant. Gen. Pharmacol. 1995, 26, 1153-1169. [CrossRef]

3. Singh, N.; Sharpley, A.L.; Emir, U.E.; Masaki, C.; Herzallah, M.M.; Gluck, M.A.; Sharp, T.; Harmer, C.J.; Vasudevan, S.R.; Cowen, P.J.; et al. Effect of the Putative Lithium Mimetic Ebselen on Brain Myo-Inositol, Sleep, and Emotional Processing in Humans. Neuropsychopharmacology 2015, 41, 1768. [CrossRef] [PubMed]

4. Romashov, L.V.; Ananikov, V.P. Self-Assembled Selenium Monolayers: From Nanotechnology to Materials Science and Adaptive Catalysis. Chem. Eur. J. 2013, 19, 17640-17660. [CrossRef] [PubMed]

5. Braga, A.; Lüdtke, D.; Vargas, F.; Braga, R. Catalytic Applications of Chiral Organoselenium Compounds in Asymmetric Synthesis. Synlett 2006, 2006, 1453-1466. [CrossRef]

6. Luo, J.; Zhu, Z.; Liu, Y.; Zhao, X. Diaryl Selenide Catalyzed Vicinal Trifluoromethylthioamination of Alkenes. Org. Lett. 2015, 17, 3620-3623. [CrossRef] [PubMed]

7. Li, M.; Guo, J.; Xue, X.S.; Cheng, J.P. Quantitative Scale for the Trifluoromethylthio Cation-Donating Ability of Electrophilic Trifluoromethylthiolating Reagents. Org. Lett. 2016, 18, 264-267. [CrossRef] [PubMed]

8. Tlili, A.; Ismalaj, E.; Glenadel, Q.; Ghiazza, C.; Billard, T. Synthetic Approaches to Trifluoromethylselenolated Compounds. Chem. Eur. J. 2018, 24, 3659-3670. [CrossRef] [PubMed]

9. Zhang, C.J. Recent Progress Toward Trifluoromethylselenolation Reactions. Chin. Chem. Soc. 2017, 64, 457-463.

10. Dale, J.W.; Emeleus, H.J.; Haszeldine, R.N. Organometallic and organometalloidal fluorine compounds. Part XIV. Trifluoromethyl derivatives of selenium. J. Chem. Soc. 1958, 2939. [CrossRef]

11. Yarovenko, N.N.; Shemanina, V.N.; Gazieva, G.B. Electrophilic trifluoromethylselenolation of terminal alkynes with Se-(trifluoromethyl)-4-methylbenzenesulfonoselenoate. Russ. J. Gen. Chem. 1959, 29, 924-927.

12. Magnier, E.; Wakselman, C. A Mild and Practical Preparation of Trifluoromethaneselenenyl Chloride. Collect. Czech. Chem. Commun. 2002, 67, 1262-1266. [CrossRef]

13. Glenadel, Q.; Ismalaj, E.; Billard, T. Benzyltrifluoromethyl (or Fluoroalkyl) Selenide: Reagent for Electrophilic Trifluoromethyl (or Fluoroalkyl) Selenolation. J. Org. Chem. 2016, 81, 8268-8275. [CrossRef] [PubMed]

14. Dong, T.; He, J.; Li, Z.-H.; Zhang, C.-P. Catalyst- and Additive-Free Trifluoromethylselenolation with [Me4N][SeCF3]. ACS Sustain. Chem. Eng. 2018, 6, 1327-1335. [CrossRef]

15. Yang, Y.; Xia, L.; Zheng, Z.; Lin, G.; Zhang, Y.; You, Y.; Weng, Z.J. $\alpha$-Trifluoromethylseleno-substituted ketones synthesis via copper-mediated trifluoromethylselenolation of $\alpha$-bromoketones. Fluor Chem. 2017, 204, 1-5. [CrossRef]

16. Glenadel, Q.; Ismalaj, E.; Billard, T. Electrophilic Trifluoromethyl- and Fluoroalkylselenolation of Organometallic Reagents. Eur. J. Org. Chem. 2017, 530-533. [CrossRef]

17. Denmark, S.E.; Rossi, S.; Webster, M.P.; Wang, H.J. Catalytic, Enantioselective Sulfenylation of Ketone-Derived Enoxysilanes. J. Am. Chem. Soc. 2014, 136, 13016-13028. [CrossRef] [PubMed] 
18. Abubakar, S.S.; Benaglia, M.; Rossi, S.; Annunziata, R. Organocatalytic $\alpha$-trifluoromethylthiolation of silylenol ethers: Batch vs continuous flow reactions. Catal. Today 2018, 308, 94-101. [CrossRef]

19. Rossi, S.; Puglisi, A.; Raimondi, L.; Benaglia, M. Synthesis of Alpha-trifluoromethylthio Carbonyl Compounds: A Survey of the Methods for the Direct Introduction of the SCF3 Group on to Organic Molecules. Chem CatChem 2018. [CrossRef]

20. The Billard group demonstrated already that such reaction could be performed and, independently from ours, simultaneously developed the $\alpha$-trifluoromethylselenolation of ketones: Ghiazza, C.; Tlili, A.; Billard, T.; Direct $\alpha-\mathrm{C}-\mathrm{H}$ Trifluoromethylselenolation of Carbonyl Compounds. Eur. J. Org. Chem. 2018, 3680-3683.

21. In the ${ }^{19} \mathrm{~F}$ NMR spectra of the crudes, few interesting peaks are present which could be potentially attributed to the desired compound, as their chemical shifts were compatible with those one could expected for trifuoromethylfunctionalized ketones based on the data we obtained in the previous experiments. We subjected the crude to purification via column chromatography, but disappointingly, we could not isolate any product. Further investigations would be needed to confirm this unexpected result.

22. The amounts of unreacted substrates, isolated products and chlorinated by-products collected were too low to account for the quantities of starting material employed These hard-to-rationalize results suggest that side reactions, leading to degradation, take place.

23. Polyisocyanurate (PIC) ferrule and zero-dead connections were employed for convenience.

24. An intense work of optimization has been performed, working with different stoichiometry of the reagents and different concentrations, but only marginal improvements of the yields were obtained in the case of ketones $\mathbf{7 b}$ and $\mathbf{7 c}$, the best results being reported in Table 2 .

25. An extensive experimental work has been done in the attempt to further improve the reaction yields; however, the use of higher concentrations of ketones was hampered by low solubility of the reagents, a crucial issue incompatible with the continuous-flow reactor set up. On the other hand, when an excess when an excess of $\mathrm{BnSeCF}_{3}$ and $\mathrm{SO}_{2} \mathrm{Cl}_{2}$ to generate $\mathrm{ClSeCF}_{3}$ was employed, the formation of significant amounts of side products, such as chlorinated derivatives, was observed, rather than an increase of the desired product yield.

Sample Availability: Samples of the compounds are available from the authors.

(c) 2019 by the authors. Licensee MDPI, Basel, Switzerland. This article is an open access article distributed under the terms and conditions of the Creative Commons Attribution (CC BY) license (http:/ / creativecommons.org/licenses/by/4.0/). 